\title{
THE INFLUENCE OF PASSENGER-DRIVER INTERACTION ON YOUNG DRIVERS
}

\author{
Ryan Toxopeus, Robert Ramkhalawansingh, \& Lana Trick \\ University of Guelph \\ Guelph, Ontario, Canada \\ Email: rtoxopeu@gmail.com
}

\begin{abstract}
Summary: The mechanisms for young drivers being at increased risk of collision with peer aged passengers in the car are not well understood. Most studies infer a link between passenger distraction and the number of passengers, but a causal link has not been previously shown. A group of young drivers with their full Ontario, Canada $G$ license were tested in a simulated driving environment in three conditions. The first condition involved a peer aged female passenger who asked the driver questions as they navigated a course. The second condition involved the same passenger sitting silently in the passenger seat while the driver navigated a course. The third condition involved the passenger being absent, and the driver was alone in the car while they navigated a course. Speed and way finding behaviours were found to deteriorate in the first condition compared to the other two, and standard deviation of lane position and reaction times were found to improve in the first condition compared to the other two, indicating that the drivers were moving their eyes around the environment less with conversation. This highlights the importance of reducing passenger distractions for younger drivers.
\end{abstract}

\section{OBJECTIVES}

Young drivers are at increased risk of injury and fatality when there are peer aged passengers in the car with them. Although this is a well documented phenomenon, the reasons for these findings are inferred, not studied. The purpose of this research is to determine to what extent passenger distraction via conversation affects driving performance. Our way finding measure is a new measure used in this context.

Traffic accidents claim 1.2 million lives each year, many of which were young people. Vehicle accidents are the leading cause of death for those between the ages of 15 and 19 and the second leading cause of death for those aged 20 to 24 (WHO, 2007). Therefore, understanding the factors that contribute to these accidents has become a major public concern.

Studies found that drivers under the age of 25 are overrepresented in terms of their collision involvement (Lam, 2003; Cooper et al., 2005; Machin \& Sankey, 2008). There are a wide range of factors that contribute to the elevated crash risk for young drivers including fatigue (Lam, 2003), excessive speed for prevailing road conditions (McKnight \& McKnight, 2003), and poor situational awareness (Borowsky et al., 2009; Lee, 2007). Research has implicated the presence of passengers as a collision risk. The majority of this research states that crash risk increases as the number of peer-aged passengers increases. The purpose of this study was to study conversation, a potential risk for passengers increasing collision rates. 
Lam (2003) demonstrated that the risk of being injured in a crash systematically increases with the number of passengers. While this pattern was present for drivers aged 16 through 24, it was most salient among drivers aged 16 to 17 who were first learning to drive. Ultimately, more than half of all deaths resulting from 16 to 17 year old drivers crashing occur when passengers younger than 20 are being transported (Williams, 2003). These studies infer a causal link between transporting teen passengers and collision risk for teen drivers.

Further investigations sought to determine what factors precipitate collisions while carrying passengers. For example, Simons-Morton et al. (2005) observed traffic leaving a high school. They tracked the age and sex of the driver along with the number, sex, and relative age of the passengers. At surrounding locations, the speed and headway of their vehicle was measured and compared to general traffic. They found the presence of a male passenger was associated with shorter headways for both male and female drivers while female passengers increased headway. Similarly, male and female drivers drove faster in the presence of teenage male passengers. Overall, teenage male drivers with teenage male passengers exhibited a rate of risky driving behaviour more than double the rate of general traffic (Simons-Morton et al., 2005).

While a number of studies have corroborated these findings, the mechanisms by which passengers increase crash risk are not well understood (Williams, 2003). The reason these mechanisms are not well understood is the majority of the research to date has focused on accident data from various authorities (Lam et al., 2003). Furthermore, there is no way of understanding the events leading up to an averted collision, nor other incidents where the police were not summoned and a subsequent report was not generated.

The current literature states that teen drivers with teen passengers are at an elevated risk for crash related injury and fatality. However, few investigations have examined what elements of their presence influence teen drivers or how these effects manifest themselves in terms of driving performance. The presence of a teenage passenger may affect the driver (Williams, 2003) but a number of studies have indicated that social influence and verbal distraction may also be noteworthy factors (Lam et al., 2003; McPhee et al., 2004; White and Caird, 2010). McPhee and colleagues (2004) used a computer search task where participants were asked to search for regulatory roadway signs while listening to short prose passages and answering questions about them. They found the conversation element did interfere with processing in that single task, but they also noted that a task such as driving requires the driver to do more than one task simultaneously, so driving performance could be degraded by conversation.

The aim of this study was to investigate the influence of passenger presence and passenger distraction in an immersive driving simulation. To do so, we compared the driving performance of teenage drivers while manipulating the dynamic between the driver and the peer-aged passenger, along with having a control condition with no passenger present. It was hypothesized that driver performance would be adversely affected by a young passenger who is actively distracting them compared to a passive occupant or driving by themselves. There are two new concepts in this study: The distracting passenger versus silent passenger manipulation and the way finding task as a measure of distraction. 


\section{METHODS}

A Drive Safety DS-600c fixed base driving simulator was used to give the participants a 300 degree wrap around immersive simulated driving experience. Three rural scenarios were created with speed limits changing between 70 and 80 kilometers per hour (kph). Each one was designed to take approximately 12 minutes to complete. For the way finding task each scenario included 6 choice points, 2 of which required the driver to make a turn. Half of the intersections had signs with city names. The names Kimball, Longmont and Ordway were used for target locations in the scenarios, and they all appeared in each scenario. The other half had landmark buildings, which were a fire hall, a hotel, and a gas station which all appeared in all three scenarios. For each scenario a set of audio-visual instructions were given to the participant with the name of a city they needed to find, along with a landmark building they needed to turn right or left at. There were also 9 hazards per scenario, with 6 hazards emerging from the right side of the road behind occluding objects, and 3 emerging from the left in a similar manner.

\section{Subjects}

Twenty participants age 17-23 $(M=19.4, S D=1.35,12$ males, 8 females $)$ took part in the study. Each person had their Full G Ontario driving license, and needed to be under the age of 25. On average, the drivers had driven within the last three weeks $(M=9.68$ days, $S D=10.19)$, drove 15.5 times per month $(S D=11.95)$ for an average of 62.25 minutes per day $(S D=67.7)$ with an estimated average distance of $33.53 \mathrm{~km}$ per day $(S D=48.36)$. They were recruited from an introductory psychology participant pool and given course credit for their participation.

\section{Testing the Passenger-Driver Interaction}

There were three conditions: In the first condition a young female research intern was in the car with the driver and asked them a series of questions (Talking), in the second condition the same research intern sat quietly in the car (Silent), and in the third condition the research intern was not present in the car (Absent). Both the driving scenarios and passenger conditions were counterbalanced to ensure there were no order effects for either scenarios or conditions. In the Talking condition drivers were asked questions about television shows and movies they enjoyed, upcoming exams, plans for holidays, brushes with celebrities, their childhood hometown, and dangerous situations they may have found themselves in. If the participant was not talkative, a variety of questions were used asking about topics such as school, travel, pets, and sports.

Way finding was analyzed by looking at missed turns (driving straight through a choice point intersection), wrong turns (turning left instead of right, or vice versa at a choice point intersection) and extra turns (turning at intersections they were not instructed to turn at).

Driving behaviours were measured by looking at the driver's speed in kph and weaving, measured using standard deviation of lane position (SDLP). Speed and weaving were recorded at a frequency of $60 \mathrm{~Hz}$. The data was taken from the beginning, middle, and end of each drive in $1 \mathrm{~km}$ increments for a total of $3 \mathrm{~km}$ per scenario. The speed limit changed throughout the scenarios, with some areas being $70 \mathrm{kph}$ and others $80 \mathrm{kph}$. Each segment of the drive used in the 
speed analysis had the average speed limit calculated, and the drivers' speeds were then compared to those averages by subtracting the speed limit from their average speeds.

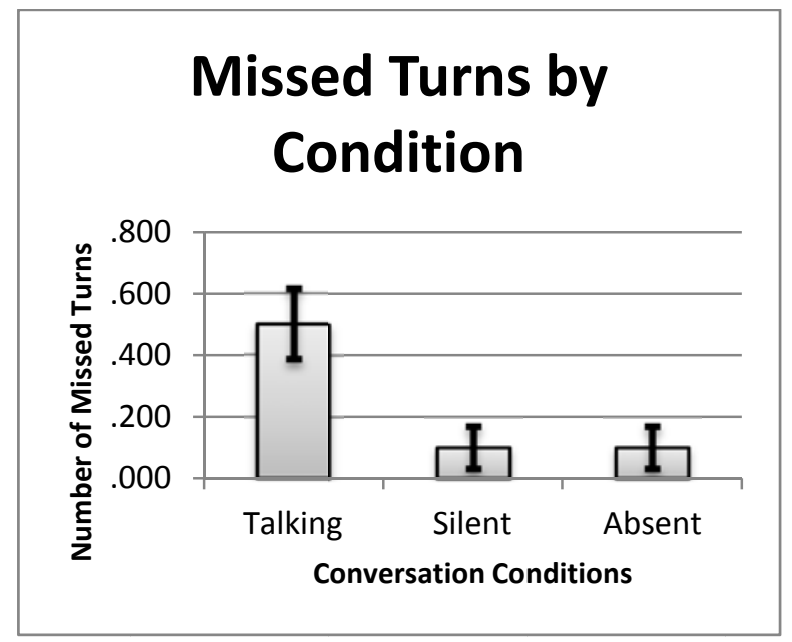

Figure 1. Drivers who were distracted by conversation with a peer aged female passenger were more likely to miss turns to get where they were supposed to go

Additionally, each drive included 9 hazards that popped into the road in front of the driver: Six appeared from the right side of the road, and three appeared from the left. Hazard reaction time (HRT) was measured as the time between when the hazard first appeared from behind an occluding object (3.5 seconds headway) to the time the participant first touched the brake. The number of collisions with hazards was measured.

\section{Analysis}

A one-way repeated-measures ANOVA was used to examine if the conditions had an effect on the dependent variables of way finding, speed, SDLP, HRT, and collisions.

\section{RESULTS}

Figure 1 shows how conversation was associated with a higher number of missed turns $(F(2,38)$ $=7.80, p=0.001)$. Drivers missed more turns when there was a passenger in the car talking to them $(M=0.50, S D=0.513)$ than when the passenger was silent $(M=0.10, S D=0.308)$ or absent $(M=0.10, S D=0.308)$. There were few wrong turns, thus although the relationship looks the same, it did not approach significance $(F(2,38)=0.241, p=0.79)$. There was no relationship found for extra turns $(F(1.34,25.40)=1.54, p=0.23)$.

When missed turns and wrong turns were summed to derive a measure of overall errors at choice points requiring the driver to make a turn, condition also had a significant effect $(F(2,38)=$ $6.44, p=0.004)$. Again drivers made the most errors when the passenger was asking them questions $(M=0.60, S D=0.598)$ when compared to the passenger remaining silent $(M=0.15$, $S D=0.366)$ or being absent from the vehicle $(M=0.15, S D=0.366)$. 

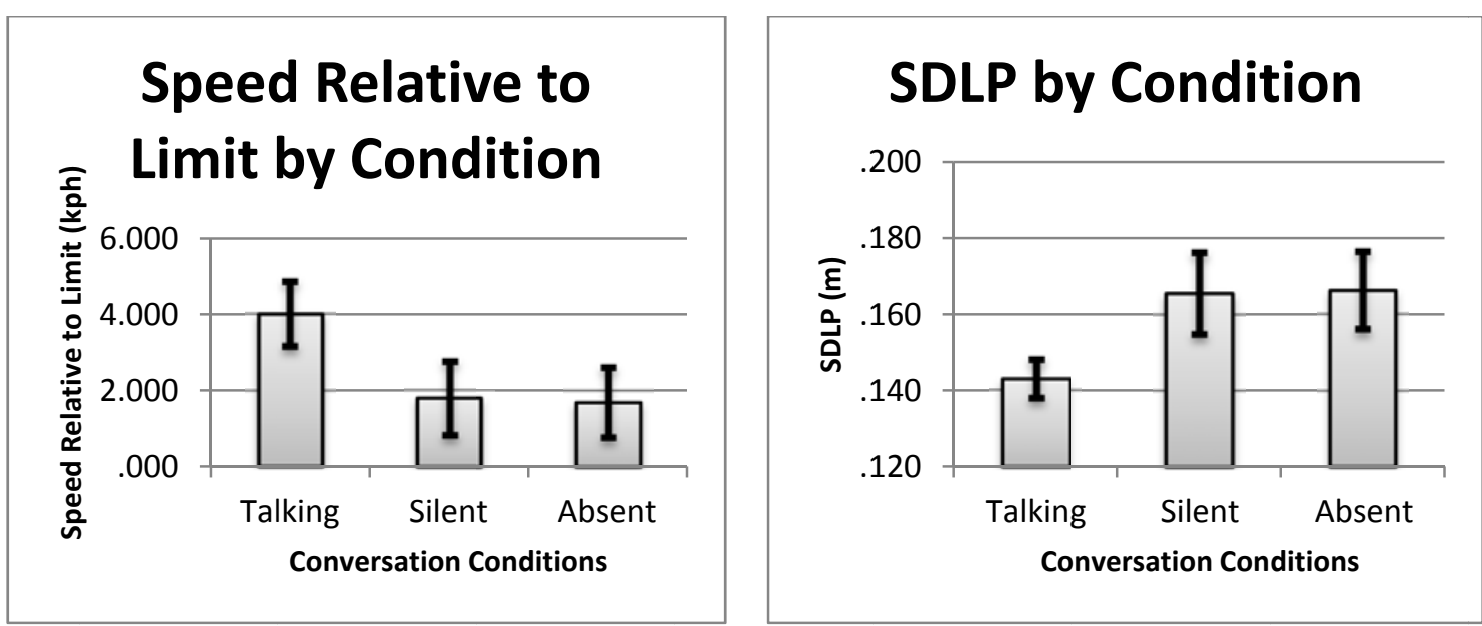

Figure 2. Speed increased when drivers were distracted by peer age female passengers compared to having a passenger quietly in the car and being alone in the car (left); SDLP decreased in the distraction condition compared to the other two (right)

Figure 2 illustrates the relationship between conversation and speed and SDLP. A significant relationship between conversation and speed emerged $(F(2,38)=7.07, p=0.002)$, where people drove faster when they had a passenger talking with them $(M=4.00 \mathrm{kph}$ over the speed limit, $S D=3.82)$ than when the passenger was silent $(M=1.79 \mathrm{kph}$ over the speed limit, $S D=$ $4.30)$ or absent $(M=1.69 \mathrm{kph}$ over the speed limit, $S D=4.11)$. However, although their speed increased, their weaving decreased $(F(2,38)=5.23, p=0.01)$ with the passenger talking to them $(M=143 \mathrm{~cm}, S D=23)$ when compared to when the passenger was silent $(M=166 \mathrm{~cm}, S D=$ 48) and absent $(M=166 \mathrm{~cm}, S D=46)$.

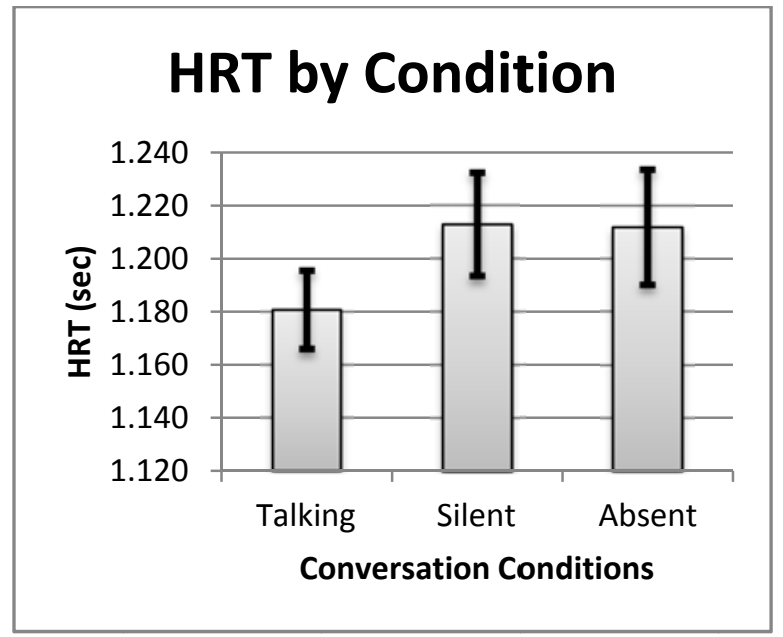

Figure 3. Drivers were faster to press the brake when they were being distracted by conversation than they were when the passenger was silent or absent

Figure 3, below, shows a trend toward significance for $\operatorname{HRT}(F(1.46,27.66)=1.96, p=0.11)$ where people were faster at reacting in the condition where the passenger was talking to them $(M=1.18 \mathrm{~s}, S D=0.066)$ than when the passenger was silent $(M=1.21 \mathrm{~s}, S D=0.087)$ or absent $(M=1.21 \mathrm{~s}, S D=0.097)$. There was no relationship between passenger condition and collisions $(F(1.46,27.69)=1.00, p=0.357)$. 


\section{CONCLUSIONS}

It is important to remember that these results were found in good driving conditions. There was only one passenger (more passengers mean greater risk (Lam, 2003)), the passenger was female (male passengers elicit more dangerous driving (Simons-Morton et al., 2005)), and the conversation was fairly mundane.

Some performance measures showed degradation with conversation in the vehicle, and others showed improvements. This mixed result may be best explained by a reduction in eye movement due to distraction (Sodhi, Reimer \& Llamazares, 2002). The authors found that drivers' eyes stopped scanning the environment with increased cognitive load, but rather wandered around a central location. Their findings help explain how drivers missed important cues for way finding and speed control, since they were not centrally located. This reduction in eye movement could put the driver and passenger(s) in danger if hazards were not located centrally.

McPhee and colleagues (2004) found that a stationary computer task which required searching for regulatory and warning road signs suffered when coupled with the task of listening to short prose passages and then answering questions about them. This decrement in search may be related to the failure to perform the wayfinding task our drivers were given.

We did not find evidence to support Williams’ (2003) idea that the presence of a teenage passenger may alone affect the driver. In fact, their driving behaviours were nearly identical with the passenger remaining silent and with no passenger in the car with them at all.

Given that younger drivers are at high risk of distraction with passenger conversation, licensing programs should include rules limiting the number and type of passengers present with young drivers. Some Canadian provinces already have such restrictions for novice drivers. In British Columbia new drivers with a learner's permit cannot have more than two passengers for their first year of driving, one of whom must be a fully licensed driver over the age of 25 . In the beginning of training, Nova Scotia, Newfoundland and Labrador are even stricter. With a learner's permit, the novice driver is only allowed to have one passenger who must be an experienced driver. However, in Newfoundland and Labrador after one year experience with the second level of license the novice driver is allowed to carry passengers during the day. In Nova Scotia the learner's permit period is only six months long, and can be reduced to three months if the driver takes a government recognized driving course. Since the results for this study were found with fully licensed young drivers in day time conditions, this issue should also be raised with policy makers for all young drivers in all provinces.

A limitation of this study was the use of only female peer aged passengers. A study by White and Caird (2010) found that there are gender interactions with conversation in vehicles, where having a passenger of the opposite gender can increase "look but failed to see" errors when the driver is attracted to the passenger. Unfortunately there were only female research interns available over both semesters that the study was run, so it might be of interest to look at the effects of a male peer aged passenger on drivers' ability to perform the same tasks. An analysis by gender was completed, but due to length restrictions could not be added here. 


\section{ACKNOWLEDGEMENTS}

The authors would like to thank Auto21, Canada Foundation for Innovation, Ontario Innovation Trust and National Sciences and Engineering Research Council for funding the research project. Additionally, we would like to thank our team of research interns who helped run participants through the study: Annette Erasmus, Leah Gordon, Alicia McNeely, Kate Gauthier, Brittany Draycott, Athena Papageorge, Kimberly Steele, and Danielle Belliveau.

\section{REFERENCES}

Borowsky, A., Oron-Gilad, T., \& Parmet, Y. (2009). Age and skill difference in classifying hazardous traffic scenes. Transportation Research Part F: Psychology and Behaviour, 12(4), 277-287.

Cooper, D., Atkins, F., \& Gillen, D. (2005). Measuring the impact of passenger restrictions on new teenage drivers. Accident Analysis and Prevention, 37(1), 19-23.

Lam, L. T. (2003). Factors associated with young drivers' car crash injury: Comparisons among learner, provisional, and full licensees. Accident Analysis and Prevention, 35(6), 913-920.

Lam, L. T., Norton, R., Woodward, M., Connor, J., \& Ameratunga, S. (2003). Passenger carriage and car crash injury: A comparison between younger and older drivers. Accident Analysis and Prevention, 35(6), 861-867.

Lee, J. D. (2007). Technology and teen drivers. Journal of Safety Research, 38(2), 203-213.

Machin, A. M., \& Sankey, K. S. (2008). Relationships between young drivers’ personality characteristics, risk perceptions, and driving behaviour. Accident Analysis and Prevention, 40(2), 541-547.

McKnight, A. J., \& McKnight, A. S. (2003). Young novice drivers: Careless or clueless? Accident Analysis and Prevention, 35(6), 921-925.

McPhee, L., Scialfa, C., Dennis, W., Ho, G., \& Caird, J. (2004). Age differences in visual search for traffic signs during a simulated conversation. Human Factors, 46(4), 674-685.

Simons-Morton, B., Lerner, N., \& Singer, J. (2005). The observed effects of teenage passengers on the risky driving behaviour of teenage drivers. Accident Analysis and Prevention, 37(6), 973-982.

Shodi, M., Reimer, B., \& Llamazares, I. (2002). Glance analysis of driver eye movements to evaluate distraction. Behaviour Research Methods, Instruments, \& Computers, 34(4), 529538.

White, C., \& Caird, J. (2010). The blind date: The effects of change blindness, passenger conversation and gender on looked-but-failed-to-see (LBFTS) errors. Accident Analysis and Prevention, 42(6), 1822-1830.

Williams, A. F. (2003). Teenage drivers: Patterns of risk. Journal of Safety Research, 34(1), 515.

World Health Organization (2007). Youth and road safety. 